\title{
Assessing tumor angiogenesis using dynamic contrast-enhanced integrated magnetic resonance-positron emission tomography in patients with non-small-cell lung cancer
}

Yu-Sen Huang ${ }^{1,2}$, Jenny Ling-Yu Chen ${ }^{1,3,4}$, Hsin-Ming Chen ${ }^{2}$, Li-Hao Yeh², Jin-Yuan Shih ${ }^{5}$, Ruoh-Fang Yen ${ }^{6}$ and Yeun-Chung Chang ${ }^{1,2^{*}}$

\begin{abstract}
Background: Angiogenesis assessment is important for personalized therapeutic intervention in patients with nonsmall-cell lung cancer (NSCLC). This study investigated whether radiologic parameters obtained by dynamic contrast-enhanced (DCE)-integrated magnetic resonance-positron emission tomography (MR-PET) could be used to quantitatively assess tumor angiogenesis in NSCLC.

Methods: This prospective cohort study included 75 patients with NSCLC who underwent DCE-integrated MR-PET at diagnosis. The following parameters were analyzed: metabolic tumor volume (MTV), maximum standardized uptake value $\left(S U V_{\text {max }}\right)$, reverse reflux rate constant $\left(k_{e p}\right)$, volume transfer constant $\left(K^{\text {trans }}\right)$, blood plasma volume fraction $\left(V_{p}\right)$, extracellular extravascular volume fraction $\left(V_{e}\right)$, apparent diffusion coefficient (ADC), and initial area under the time-to-signal intensity curve at $60 \mathrm{~s}$ post enhancement $\left(\mathrm{iAUC}_{60}\right)$. Serum biomarkers of tumor angiogenesis, including vascular endothelial growth factor-A (VEGF-A), angiogenin, and angiopoietin-1, were measured by enzyme-linked immunosorbent assays simultaneously.

(Continued on next page)
\end{abstract}

\footnotetext{
* Correspondence: ycc5566@ntu.edu.tw

'Department of Radiology, National Taiwan University College of Medicine, No. 7, Chung-Shan S. Rd., Taipei 100, Taiwan

${ }^{2}$ Department of Medical Imaging, National Taiwan University Hospital, Taipei,

Taiwan

Full list of author information is available at the end of the article
}

(c) The Author(s). 2021 Open Access This article is licensed under a Creative Commons Attribution 4.0 International License, which permits use, sharing, adaptation, distribution and reproduction in any medium or format, as long as you give appropriate credit to the original author(s) and the source, provide a link to the Creative Commons licence, and indicate if changes were made. The images or other third party material in this article are included in the article's Creative Commons licence, unless indicated otherwise in a credit line to the material. If material is not included in the article's Creative Commons licence and your intended use is not permitted by statutory regulation or exceeds the permitted use, you will need to obtain permission directly from the copyright holder. To view a copy of this licence, visit http://creativecommons.org/licenses/by/4.0/ The Creative Commons Public Domain Dedication waiver (http://creativecommons.org/publicdomain/zero/1.0/) applies to the data made available in this article, unless otherwise stated in a credit line to the data. 
(Continued from previous page)

Results: Serum VEGF-A ( $p=0.002)$, angiogenin ( $p=0.023)$, and Ang-1 $(p<0.001)$ concentrations were significantly elevated in NSCLC patients compared with healthy individuals. MR-PET parameters, including MTV, $K^{\text {trans }}$, and $k_{\text {ep }}$, showed strong linear correlations $(p<0.001)$ with serum angiogenesis-related biomarkers. Serum VEGF-A concentrations $(p=0.004)$, MTV values $(p<0.001)$, and $k_{\text {ep }}$ values $(p=0.029)$ were significantly higher in patients with advanced-stage disease (stage III or IV) than in those with early-stage disease (stage I or II). Patients with initial higher values of angiogenesis-related MR-PET parameters, including MTV $>30 \mathrm{~cm}^{3}(p=0.046), K^{\text {trans }}>20010^{-3} / \mathrm{min}$ $(p=0.069)$, and $k_{\mathrm{ep}}>90010^{-3} / \mathrm{min}(p=0.048)$, may have benefited from angiogenesis inhibitor therapy, which thus led to significantly longer overall survival.

Conclusions: The present findings suggest that DCE-integrated MR-PET provides a reliable, non-invasive, quantitative assessment of tumor angiogenesis; can guide the use of angiogenesis inhibitors toward longer survival; and will play an important role in the personalized treatment of NSCLC.

Keywords: Personalized medicine, Radiologic biomarkers, Angiogenesis inhibitors, Survival

\section{Background}

Non-small-cell lung cancer (NSCLC) is characterized by poor prognosis and is the leading cause of cancerrelated mortality worldwide, and tumor angiogenesis pathways are essential in the process of primary tumor growth, proliferation, and development of distant metastases. Therefore, targeted therapy against angiogenesis has been identified as an important strategy and has now been clinically approved for the first-line treatment of NSCLC in selected patients [1, 2].

Tumor angiogenesis in NSCLC can be noninvasively identified by imaging techniques [3]. Dynamic contrast-enhanced (DCE)-integrated magnetic resonance imaging (MRI) uses permeability and perfusion parameters that are essential in the assessment of tumor angiogenesis and aggressiveness [4]; common DCE-MRI imaging protocols and analysis methods are an important tool in both preclinical and clinical research [5]. ${ }^{18}$ Fluoro-2- deoxyglucose- positron emission tomography (FDG-PET) assesses intratumoral glucose metabolism with parameters associated with tumor angiogenesis, including the maximum standardized uptake value $\left(\mathrm{SUV}_{\max }\right)$ and metabolic tumor volume (MTV), thereby providing biological and physiological information about tumor viability [6-8]. Both PET and MRI bear potential for non-invasive assessment, and hybrid PET/MR imaging may be suitable for precise evaluation. DCEintegratedMR-PET combines the advantages of PET metabolic analysis and MRI permeability imaging for an integral evaluation and thus provides a powerful non-invasive imaging technology to assess tumor biology.

In addition, tumor angiogenesis can also be indirectly evaluated by serum analysis. Serum vascular endothelial growth factor (VEGF)-A, angiogenin, and angiopoietin-1 (Ang-1), which are secreted by tumors in the body and thus indicate total tumor growth and aggressiveness, are considered biomarkers for tumor angiogenesis [911].

Given that tumor angiogenesis, an important prognostic indicator in NSCLC, can be evaluated by both imaging and serum analyses [12, 13], we investigated whether radiologic parameters derived from DCEintegratedMR-PET scans correlate with serum angiogenesis-related biomarkers. The present study could provide a non-invasive method for the quantitative assessment of tumor angiogenesis in NSCLC and might guide the use of angiogenesis inhibitors in clinical practice.

\section{Methods}

\section{Study design and patient enrolment}

This prospective study was conducted according to the guidelines of the Declaration of Helsinki and its later amendments, and was approved by the National Taiwan University Hospital Research Ethics Committee (approval number: 201712101RIND). Written informed consent was obtained from all participants after the nature of the procedures had been fully explained.

Patients with a new diagnosis of NSCLC were enrolled, and they underwent DCE-integratedMR-PET scans before treatment and were staged using the American Joint Committee on Cancer staging manual (8th edition) [14]. Histopathological reviews were carried out by an experienced pathologist who majored in thoracic oncology. For patients with adenocarcinoma, cancer specimens were analyzed using RNA reverse transcriptionpolymerase chain reaction or direct DNA sequencing, as previously described [15]. Epidermal growth factor receptor (EGFR) mutation was defined as the presence of an EGFR exon19del or L858R mutation in tumor genomic DNA, and anaplastic lymphoma kinase (ALK)/c- 
Table 1 Patient clinical characteristics $(n=75)$

\begin{tabular}{|c|c|c|}
\hline & $\mathrm{n}$ & $\%$ \\
\hline \multicolumn{3}{|l|}{ Age (years) } \\
\hline Median (range) & $65(40-80)$ & \\
\hline \multicolumn{3}{|l|}{ Sex } \\
\hline Male & 49 & 65 \\
\hline Female & 26 & 35 \\
\hline \multicolumn{3}{|l|}{ Stage grouping* } \\
\hline । & 12 & 16 \\
\hline$\|$ & 5 & 7 \\
\hline III & 31 & 41 \\
\hline IV & 27 & 36 \\
\hline \multicolumn{3}{|l|}{ Histology } \\
\hline Adenocarcinoma & 56 & 75 \\
\hline Squamous cell carcinoma & 18 & 24 \\
\hline Pleomorphic carcinoma & 1 & 1 \\
\hline \multicolumn{3}{|l|}{ EGFR status ${ }^{\#}$} \\
\hline Mutation not found & 47 & 63 \\
\hline Mutation found & 28 & 37 \\
\hline \multicolumn{3}{|l|}{ ALK/ROS1 status $^{\dagger}$} \\
\hline Rearrangement not found & 72 & 96 \\
\hline Rearrangement found & 3 & 4 \\
\hline
\end{tabular}

Abbreviations: $\mathrm{EGFR}=$ epidermal growth factor receptor; $\mathrm{ALK}=$ anaplastic lymphoma kinase; ROS1 = c-ros oncogene 1

* Staging was performed according to the guidelines of the American Joint Committee on Cancer, 8th edition

\# EGFR mutation was defined as the presence of an EGFR exon19del or L858R mutation in tumor genomic DNA

† ALK/ROS1 rearrangement was defined as the presence of an ALK or ROS1 rearrangement in tumor genomic DNA

ros oncogene 1 (ROS1) rearrangement was defined as the occurrence of an ALK or ROS1 rearrangement in tumor genomic DNA. A total of 15 healthy individuals (median age, 55 years; range, $33-70$ years; 5 women and 10 men) served as healthy controls.

\section{Magnetic resonance-positron emission tomography examinations}

The simultaneous 3-TMR-PET machine (Biograph mMR; Siemens Healthcare, Erlangen, Germany) was used to perform MR-PET imaging, as previously described $[4,16]$. The examination time was approximately one hour. For obtaining positron emission tomography images, intravenous administration of $3.3 \mathrm{MBq}$ of FDG per $\mathrm{kg}$ body weight (range, 132-300 MBq) was performed on all patients after they were made to fast; images were obtained at $60 \mathrm{~min}$ after injection; a 4-min scan with $864-1335 \mathrm{~mm}$ in length was applied for one bed position, to a total of five bed positions with a total acquisition time of $20 \mathrm{~min}$ per patient, per standard protocol for whole-body PET images. For image reconstruction, the ordered-subsetexpectation-maximization iterative algorithm with a 5-mmpost-reconstruction Gaussian filter was used.

For obtaining magnetic resonance images, a contrast agent (gadobutrol [0.2 mmol $/ \mathrm{kg}]$; Gadavist, Bayer Healthcare, Berlin, Germany) was intravenously administered. Axial volumetric interpolated breath-hold examination sequence was performed with parameters as follows: repetition time [TR]/echo time [TE] 4.03/1.45 $\mathrm{ms}$; slice thickness $2 \mathrm{~mm}$; field of view $320 \times 260 \mathrm{~mm}$; fractional anisotropy (FA) $9^{\circ}$; matrix size $320 \times 250$; number of excitations (NEX) 1; image acquisition time $95 \mathrm{~s}$ in five divided scans. The monoexponential function (b-values: 50, 200, 600, and $1000 \mathrm{~s} / \mathrm{mm}^{2}$ ) was used to construct ADC maps.

\section{Magnetic resonance-positron emission tomography image analysis}

The two-compartment Tofts model and after-motion registration were employed for pharmacodynamic analyses of magnetic resonance images. Two observers (two radiologists who specialized in chest imaging, with 8 and 22 years of experience in chest MRI, respectively) drew freehand the regions of interest in consensus. Image parameters of the largest primary lung tumor seen on T1weighted MRI (contrast-enhanced) were evaluated using a commercial software (MIStar; Apollo Medical Imaging Technology, Melbourne, Australia): reverse reflux rate constant $\left(k_{\mathrm{ep}}\right)$, volume transfer constant $\left(K^{\text {trans }}\right)$, blood plasma volume fraction $\left(v_{p}\right)$, extracellular extravascular volume fraction $\left(v_{e}\right)$, apparent diffusion coefficient (ADC), and initial area under the time-to-signal intensity curve at $60 \mathrm{~s}$ post enhancement $\left(\mathrm{iAUC}_{60}\right)$. ADC indicates the amount of water diffusion within tumors; $K^{\text {trans }}$ evaluates the diffusive transport of low-molecular-weight gadolinium chelates across the capillary endothelium; and $k_{\mathrm{ep}}, v_{p}$, and $v_{e}$, which indicate the reflux rate constant, plasma volume, and extracellular volume, respectively, are regarded as biomarkers predictive of tumor angiogenesis. The MTV was defined as the sum of the volumes of the primary lung tumor and the regional lymphadenopathy using a threshold of $40 \%$ of the maximal SUV, assessed through semi-automatically conducted 3-dimensional outlining by the commercial software (Syngo.via; Siemens Healthcare, Erlangen, Germany) $[6,16]$. The median total time of image analysis for each set of images was $45 \mathrm{~min}$ (range, 30-60 $\min )$.

\section{Quantification of serum angiogenic biomarkers in patients with NSCLC}

Serum samples were collected before DCEintegratedMR-PET scans. A $10-\mathrm{mL}$ blood sample was drawn from each patient, placed in vacutainer redtopped tubes (Becton Dickinson and Company, New 
(a) MR-PET axial

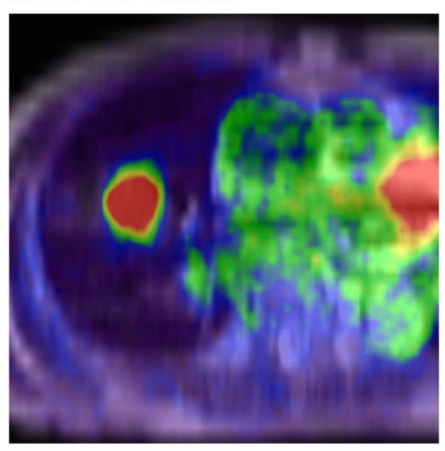

(c) T1-weighted

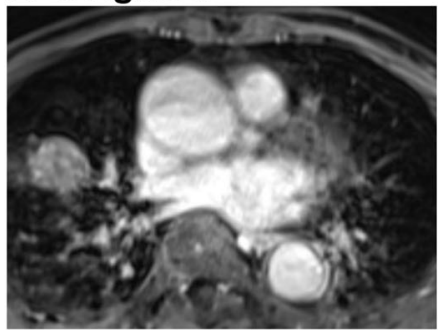

(e) $K^{\text {trans }}$ map

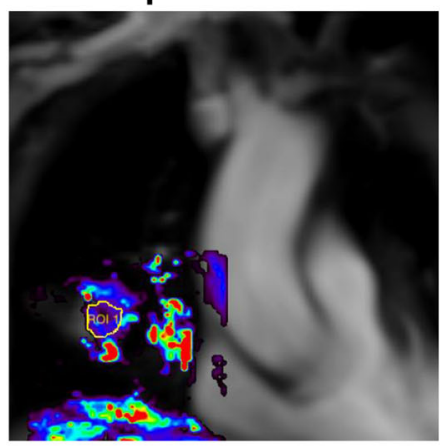

(g) $v_{e}$ map

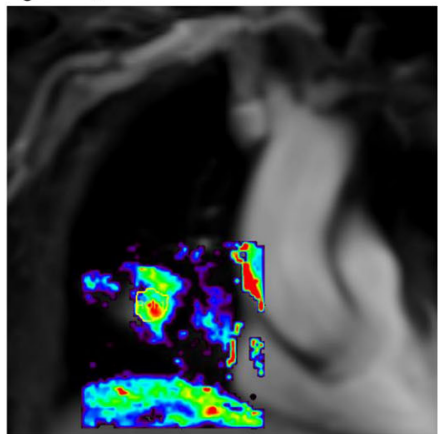

(b) MR-PET coronal

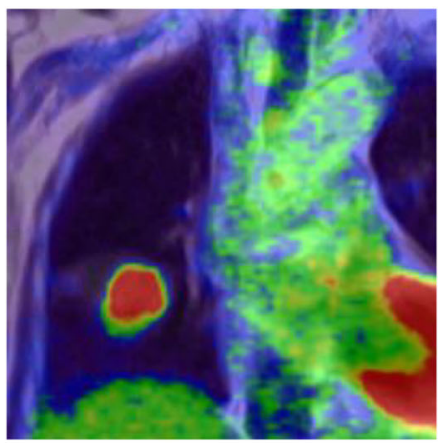

(d) ADC map

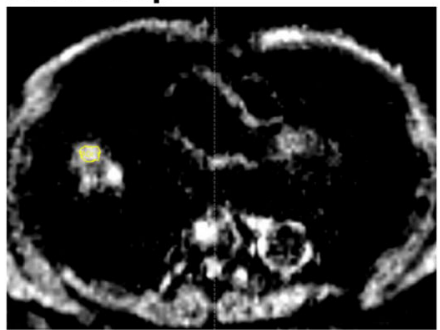

(f) $k_{\text {ep }}$ map

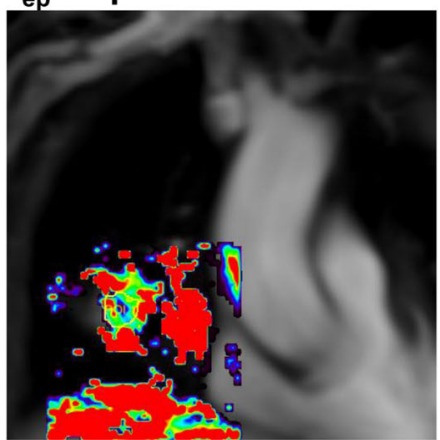

(h) $v_{p}$ map

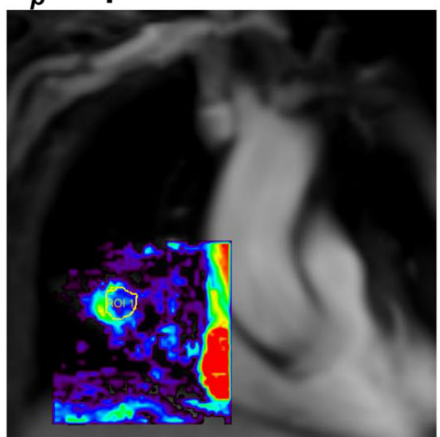

Fig. 1 Dynamic contrast-enhanced integrated magnetic resonance-positron emission tomography parameters obtained at diagnosis in a patient with right lower lung adenocarcinoma (CT2aNOMO, stage IB). Measured magnetic resonance-positron emission tomography (MR-PET) parameters were as follows: (a, b) SUV max, 11; (c) tumor size, $3.0 \mathrm{~cm}$ (by T1-weighted post contrast); (d) ADC, $1025\left(10^{-6} \mathrm{~mm}^{2} / \mathrm{s}\right) ;(\mathbf{e}) \mathrm{K}^{\text {trans }}, 110\left(10^{-3} / \mathrm{min}\right) ;(\mathbf{f})$ $k_{\mathrm{ep}}, 598\left(10^{-3} / \mathrm{min}\right) ;(\mathbf{g}) v_{e}, 202\left(10^{-3}\right) ;(\mathbf{h}) v_{p}, 107\left(10^{-3}\right)$; and $\mathrm{IAUC}_{60}, 253\left(10^{-3}\right)$. Measured serum angiogenesis-related biomarkers included VEGFA $(119 \mathrm{pg} / \mathrm{mL})$, angiopoietin-1 $(46 \mathrm{ng} / \mathrm{mL})$, angiopoietin-2 $(128 \mathrm{pg} / \mathrm{mL})$, and angiogenin $(632 \mathrm{ng} / \mathrm{mL})$. Due to medically inoperable status, the patient underwent stereotactic body radiation therapy. The patient achieved a complete response and remained disease-free for 48 months 


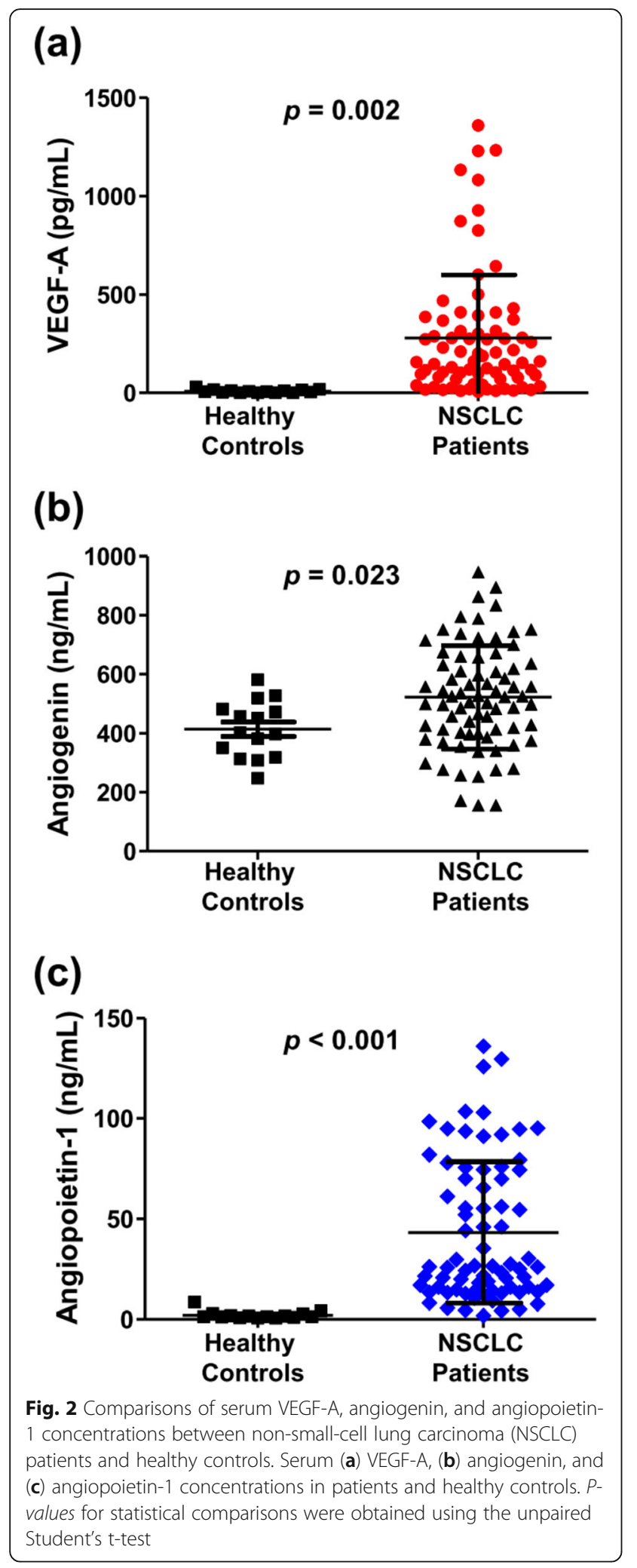

Jersey, USA), allowed to clot at room temperature for $30 \mathrm{~min}$, and then centrifuged at $1140 \times g(2500 \mathrm{rpm})$ for $30 \mathrm{~min}$. Serum was aliquoted and stored aseptically at $80^{\circ} \mathrm{C}$ until analysis. Quantitative serum angiogenic biomarker enzyme-linked immunosorbent assay kits (R\&D Systems, Minneapolis, USA) were used to measure VEGF-A, Ang-1, and angiogenin concentrations in the serum samples [17].

\section{Treatments and follow-up}

Treatment was performed according to the institutional guideline for NSCLC. The use of surgery, radiotherapy, chemotherapy, immunotherapy, targeted agents, or clinical trial enrolment was recommended by the institutional multidisciplinary lung cancer panel discussion to improve patients' clinical outcomes. Angiogenesis inhibitors approved by the Taiwan Food and Drug Administration for the treatment of selected NSCLC patients, including bevacizumab and ramucirumab, were administered at the discretion of the attending physician.

Patients underwent chest radiography every $2-4$ weeks and computed tomography (CT) of the brain and chest (including the liver and adrenal glands) every 2-3 months as routine clinical practice, and other imaging studies, including PET or MRI, were conducted when necessary. Overall survival (OS) was calculated as the time from the start of any treatment until death from any cause. The median follow-up period was 27 months for surviving patients (range, 2-56 months).

\section{Statistical analyses}

The Statistical Package for Social Sciences for Windows, version 17.0 (SPSS, Chicago, IL, USA) was used for statistical analyses. Statistical comparisons of serum VEGFA, Ang-1, and angiogenin concentrations between patients and healthy controls were performed using unpaired Student's t-tests. The correlation between DCEintegratedMR-PET parameters and serum angiogenesisrelated biomarkers was evaluated by Pearson's linear regression analysis. Statistical comparisons of MR-PETderived parameters between clinical groups (male vs. female, advanced vs. early stage, adenocarcinoma vs. nonadenocarcinoma, EGFR mutation found vs. not found, and ALK/ROS1 rearrangement found vs. not found) were performed using unpaired Student's t-tests. When Bonferroni correction was applied for multiple comparisons (a total of eight MR-PET derived parameters), $p$ values less than 0.006 , a threshold obtained by dividing 0.05 by the number of tests (eight), were considered statistically significant [18]. Survival analyses were conducted using the data available on July 31, 2020. Kaplan-Meier life-table analyses were used to assess survival rates, and log-rank tests were used for prognostic parameter evaluations. 
Table 2 Correlation between dynamic contrast-enhanced integrated MR-PET parameters and serum angiogenesis-related biomarkers

\begin{tabular}{|c|c|c|c|c|c|c|c|c|c|}
\hline & \multicolumn{3}{|c|}{ VEGF-A (pg/mL) } & \multicolumn{3}{|c|}{ Angiogenin $(\mathrm{ng} / \mathrm{mL})$} & \multicolumn{3}{|c|}{ Angiopoietin-1 (ng/mL) } \\
\hline & $R^{2}$ & Slope & $p$-value* & $R^{2}$ & Slope & $p$-value* & $\overline{R^{2}}$ & Slope & $p$-value* \\
\hline $\operatorname{MTV}\left(\mathrm{cm}^{3}\right)$ & 0.16 & 2.26 & $<0.001^{+}$ & 0.22 & 1.45 & $<0.001^{\dagger}$ & 0.22 & 0.29 & $<0.001^{\dagger}$ \\
\hline$S U V_{\max }$ & 0.09 & 12.67 & 0.010 & 0.05 & 5.34 & 0.049 & 0.04 & 0.96 & 0.078 \\
\hline $\operatorname{ADC}\left(10^{-6} \mathrm{~mm}^{2} / \mathrm{sec}\right)$ & 0.03 & -0.16 & 0.184 & 0.08 & -0.16 & 0.015 & 0.11 & -0.04 & $0.004^{t}$ \\
\hline$K^{\text {trans }}\left(10^{-3} \mathrm{~min}^{-1}\right)$ & 0.14 & 0.24 & $0.001^{+}$ & 0.11 & 0.07 & $0.004^{\dagger}$ & 0.26 & 0.04 & $<0.001^{+}$ \\
\hline$k_{\text {ep }}\left(10^{-3} \min ^{-1}\right)$ & 0.31 & 0.13 & $<0.001^{+}$ & 0.20 & 0.06 & $<0.001^{t}$ & 0.21 & 0.01 & $<0.001^{\dagger}$ \\
\hline$v_{e}\left(10^{-3}\right)$ & $<0.01$ & 0.04 & 0.845 & 0.01 & -0.11 & 0.384 & 0.12 & 0.07 & $0.002^{+}$ \\
\hline$v_{p}\left(10^{-3}\right)$ & 0.05 & 0.81 & 0.066 & $<0.01$ & 0.06 & 0.790 & 0.09 & 0.13 & 0.008 \\
\hline $\operatorname{iAUC}_{60}\left(10^{-3}\right)$ & 0.02 & 0.13 & 0.221 & $<0.01$ & 0.01 & 0.926 & 0.20 & 0.05 & $<0.001^{\dagger}$ \\
\hline
\end{tabular}

Abbreviations: MR-PET = magnetic resonance-positron emission tomography; VEGF = vascular endothelial growth factor; $M$ TV $=$ metabolic tumor volume; $S U V_{\text {max }}=$ maximum standardized uptake value; $k_{\mathrm{ep}}=$ reverse reflux rate constant; $K^{\text {trans }}=$ volume transfer constant; $v_{p}=$ blood plasma volume fraction; $v_{e}=$ extracellular extravascular volume fraction; $A D C=$ apparent diffusion coefficient; iAUC 60 initial area under the time-to-signal intensity curve at $60 \mathrm{~s}$ post enhancement * Significance tested using Pearson's linear regression analysis

${ }^{\dagger}$ When Bonferroni correction was applied for multiple comparisons (a total of eight MR-PET derived parameters), $p$-values $<0.006$ were considered statistically significant

\section{Results}

\section{Patient characteristics}

Between March 2017 and December 2018, 75 patients newly diagnosed with NSCLC (26 women, 49 men; median age: 65 years; range: $40-80$ years; Table 1 ) were prospectively enrolled. A patient's DCE-integratedMR-PET images are shown in Fig. 1. Fifty-six tumors $(75 \%)$ were adenocarcinomas, 18 (24\%) were squamous cell carcinomas, and one (1\%) was a pleomorphic carcinoma. More than one-third of the patients $(36 \%)$ had stage IV disease at diagnosis. Tumor genomic testing revealed that 28 patients (37\%) carried an EGFR mutation, and three (4\%) had an ALK/ROS1 rearrangement. Serum VEGF-A (280 $\mathrm{pg} / \mathrm{mL}$ in NSCLC patients vs. $9 \mathrm{pg} / \mathrm{mL}$ in healthy controls; $p=0.002)$, angiogenin $(522 \mathrm{ng} / \mathrm{mL}$ in NSCLC patients vs. $414 \mathrm{ng} / \mathrm{mL}$ in healthy controls; $p=0.023$ ), and Ang-1 (mean: $43 \mathrm{ng} / \mathrm{mL}$ in NSCLC patients vs. $2 \mathrm{ng} / \mathrm{mL}$ in healthy controls; $p<0.001$ ) concentrations were significantly elevated in NSCLC patients compared with healthy individuals (Fig. 2).

\section{Correlations of MR-PET parameters with serum angiogenesis-related biomarkers}

The associations between eight MR-PET parameters and three serum angiogenesis-related biomarkers were evaluated (Table 2). When Bonferroni correction was applied for multiple comparisons (a total of eight MR-PET derived parameters), $p$-values $<0.006$, a threshold obtained by dividing 0.05 by the number of tests (eight), were considered statistically significant. As shown in Fig. 3, three MR-PET measures, namely MTV, $K^{\text {trans }}$, and $k_{\text {ep }}$, showed strong linear correlations $(p<0.001)$ with all the tested serum angiogenesis-related biomarkers, i.e., VEGF-A, angiogenin, and Ang-1, indicating that quantification MR-
PET measurement reflected the concentrations of serum angiogenesis-related biomarkers. Three other MR-PET measures, i.e., $\operatorname{ADC~}(p=0.004), v_{e} \quad(p=$ $0.002)$, and iAUC $_{60}(p<0.001)$, showed significant correlations with Ang-1, one of the tested angiogenesis-related biomarkers.

\section{MR-PET parameters and serum angiogenesis-related biomarkers in patients with clinically advanced disease} We evaluated the association of MR-PET measures and serum biomarkers with clinical tumor characteristics (Table 3). As shown in Fig. 4, serum VEGF-A concentrations $(318 \pm 349$ vs. $148 \pm 138 \mathrm{pg} / \mathrm{mL}, p=0.004)$, MTV values ( $57 \pm 61$ vs. $\left.12 \pm 17 \mathrm{~cm}^{3}, p<0.001\right)$, and $k_{\mathrm{ep}}$ values $\left(1455 \pm 779\right.$ vs. $\left.935 \pm 53210^{-3} / \mathrm{min}, p=0.029\right)$ were significantly higher in patients with advanced disease (stage III or IV) than in those with early-stage disease (stage I or II). No significant correlations were found between MR-PET or serum measures and other clinical characteristics, including sex, histology, EGFR mutation, or ALK/ROS1 rearrangement.

\section{Potential of MR-PET parameters in predicting the efficacy of angiogenesis inhibitors}

During the median follow-up period of 27 months (range, 2-56 months), 11 of the 58 patients with advanced disease (stage III or IV) received angiogenesis inhibitors (bevacizumab or ramucirumab) as part of their treatment. The survival of advanced-stage patients, grouped by initial angiogenesis-relatedMR-PET parameters, was investigated (Table 4). The grouping was based on median values of MTV, $K^{\text {trans }}$, or $k_{\text {ep }}$ in patients with advanced disease. In advanced-stage patients with initial higher angiogenesis-relatedMR-PET parameters (Fig. 5), 

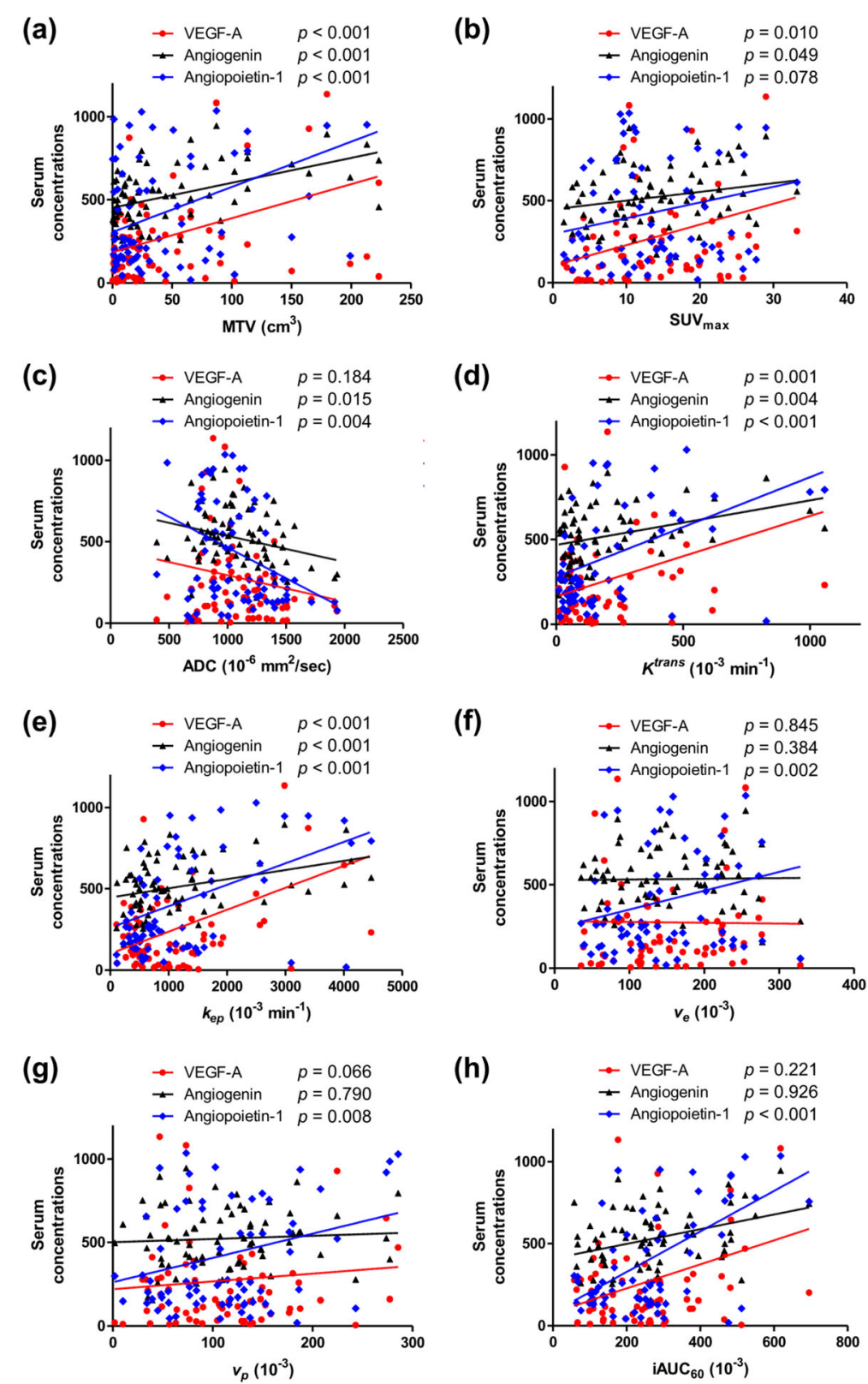

Fig. 3 Relationships between MR-PET parameters and serum angiogenesis-related biomarkers in patients with non-small-cell lung carcinoma. Scatterplots demonstrating the relationships between serum VEGF-A $(\mathrm{pg} / \mathrm{mL})$, angiogenin $(\mathrm{ng} / \mathrm{mL})$, and angiopoietin-1 $\left(10^{-1} \mathrm{ng} / \mathrm{ml}\right)$

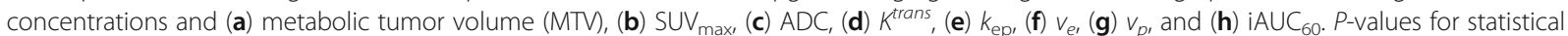
comparisons between MR-PET parameters and serum angiogenesis-related biomarkers were obtained using Pearson's linear regression analysis. When Bonferroni correction was applied for multiple comparisons (a total of eight MR-PET derived parameters), $p$-values $<0.006$ were considered statistically significant

including MTV $>30 \mathrm{~cm}^{3}(p=0.046), K^{\text {trans }}>20010^{-3} /$ $\min (p=0.069)$, and $k_{\mathrm{ep}}>90010^{-3} / \mathrm{min}(p=0.048)$, a significantly longer OS was seen when angiogenesis inhibitors were administered. However, no significant survival difference was found when angiogenesis inhibitors were administered in patients with lower initial MTV, $K^{\text {trans }}$, or $k_{\text {ep }}$.

\section{Discussion}

This is the first study, to our knowledge, to investigate correlations between DCE-integratedMR-PET imaging and serum biomarkers quantitatively assessing tumor angiogenesis in NSCLC patients. Importantly, we found that MTV, $K^{\text {trans }}$, and $k_{\text {ep }}$ values showed significant and positive correlations with serum angiogenesis-related 
Table 3 Correlations between angiogenesis-related biomarkers and clinical tumor characteristics $(n=75)$

\begin{tabular}{|c|c|c|c|c|c|c|c|c|c|c|}
\hline \multirow{2}{*}{$\begin{array}{l}\text { (mean } \pm \text { standard } \\
\text { deviation) } \\
p \text {-value }{ }^{※}\end{array}$} & \multicolumn{2}{|l|}{ Sex } & \multicolumn{2}{|c|}{ Tumor stage* } & \multicolumn{2}{|l|}{ Histology } & \multicolumn{2}{|c|}{ EGFR Mutation ${ }^{\#}$} & \multicolumn{2}{|c|}{$\begin{array}{l}\text { ALK/ROS1 } \\
\text { Rearrangement }^{\dagger}\end{array}$} \\
\hline & Male & Female & $\begin{array}{l}\text { Stage I } \\
\text { / II }\end{array}$ & $\begin{array}{l}\text { Stage III / } \\
\text { IV }\end{array}$ & Adenocarcinoma & $\begin{array}{l}\text { Non- } \\
\text { Adenocarcinoma }\end{array}$ & Found & $\begin{array}{l}\text { Not } \\
\text { found }\end{array}$ & Found & $\begin{array}{l}\text { Not } \\
\text { found }\end{array}$ \\
\hline \multirow[t]{2}{*}{ VEGF-A (pg/mL) } & $326 \pm 353$ & $\begin{array}{l}192 \pm \\
231\end{array}$ & $\begin{array}{l}148 \pm \\
138\end{array}$ & $318 \pm 349$ & $268 \pm 309$ & $313 \pm 361$ & $\begin{array}{l}254 \pm \\
317\end{array}$ & $\begin{array}{l}293 \pm \\
326\end{array}$ & $\begin{array}{l}389 \pm \\
601\end{array}$ & $\begin{array}{l}275 \pm \\
311\end{array}$ \\
\hline & 0.053 & & $0.004^{\ddagger}$ & & 0.632 & & 0.621 & & 0.774 & \\
\hline \multirow[t]{2}{*}{$\begin{array}{l}\text { Angiogenin (ng/ } \\
\mathrm{mL} \text { ) }\end{array}$} & $539 \pm 172$ & $\begin{array}{l}491 \pm \\
180\end{array}$ & $\begin{array}{l}473 \pm \\
111\end{array}$ & $537 \pm 188$ & $504 \pm 178$ & $577 \pm 160$ & $\begin{array}{l}430 \pm \\
161\end{array}$ & $\begin{array}{l}571 \pm 1 \\
64\end{array}$ & $\begin{array}{l}735 \pm \\
203\end{array}$ & $\begin{array}{l}513 \pm \\
170\end{array}$ \\
\hline & 0.174 & & 0.091 & & 0.102 & & 0.061 & & 0.196 & \\
\hline \multirow{2}{*}{$\begin{array}{l}\text { Angiopoietin-1 } \\
(\mathrm{ng} / \mathrm{mL})\end{array}$} & $49 \pm 39$ & $32 \pm 23$ & $43 \pm 31$ & $44 \pm 37$ & $38 \pm 31$ & $57 \pm 44$ & $35 \pm 30$ & $47 \pm 37$ & $53 \pm 44$ & $43 \pm 35$ \\
\hline & 0.055 & & 0.931 & & 0.095 & & 0.139 & & 0.739 & \\
\hline \multirow[t]{2}{*}{$\operatorname{MTV}\left(\mathrm{cm}^{3}\right)$} & $56 \pm 61$ & $30 \pm 46$ & $12 \pm 17$ & $57 \pm 61$ & $30 \pm 38$ & $96 \pm 74$ & $32 \pm 37$ & $60 \pm 62$ & $82 \pm 71$ & $45 \pm 57$ \\
\hline & 0.065 & & $<0.001^{\ddagger}$ & & 0.108 & & 0.139 & & 0.469 & \\
\hline \multirow[t]{2}{*}{$S U V_{\max }$} & $15 \pm 8$ & $12 \pm 6$ & $9 \pm 6$ & $15 \pm 7$ & $13 \pm 7$ & $18 \pm 8$ & $12 \pm 7$ & $15 \pm 7$ & $10 \pm 6$ & $14 \pm 8$ \\
\hline & 0.070 & & 0.101 & & 0.112 & & 0.156 & & 0.355 & \\
\hline \multirow[t]{2}{*}{$\begin{array}{l}\text { ADC }\left(10^{-6} \mathrm{~mm}^{2} /\right. \\
\mathrm{sec})\end{array}$} & $\begin{array}{l}1056 \pm \\
296\end{array}$ & $\begin{array}{l}1137 \pm \\
324\end{array}$ & $\begin{array}{l}1145 \pm \\
499\end{array}$ & $876 \pm 236$ & $1122 \pm 321$ & $975 \pm 231$ & $\begin{array}{l}1077 \pm \\
321\end{array}$ & $\begin{array}{l}1088 \pm \\
301\end{array}$ & $\begin{array}{l}1064 \pm \\
375\end{array}$ & $\begin{array}{l}1085 \pm \\
306\end{array}$ \\
\hline & 0.293 & & 0.190 & & 0.059 & & 0.883 & & 0.931 & \\
\hline \multirow[t]{2}{*}{$K^{\text {trans }}\left(10^{-3} \mathrm{~min}^{-1}\right)$} & $392 \pm 556$ & $\begin{array}{l}197 \pm \\
333\end{array}$ & $\begin{array}{l}283 \pm \\
430\end{array}$ & $464 \pm 675$ & $332 \pm 556$ & $302 \pm 265$ & $\begin{array}{l}401 \pm \\
676\end{array}$ & $\begin{array}{l}284 \pm \\
371\end{array}$ & $\begin{array}{l}633 \pm \\
1011\end{array}$ & $\begin{array}{l}312 \pm \\
474\end{array}$ \\
\hline & 0.063 & & 0.310 & & 0.754 & & 0.420 & & 0.638 & \\
\hline \multirow[t]{2}{*}{$k_{\mathrm{ep}}\left(10^{-3} \mathrm{~min}^{-1}\right)$} & $\begin{array}{l}1484 \pm \\
1350\end{array}$ & $\begin{array}{l}1062 \pm \\
1300\end{array}$ & $\begin{array}{l}935 \pm \\
532\end{array}$ & $\begin{array}{l}1455 \pm \\
779\end{array}$ & $1211 \pm 1337$ & $1710 \pm 1310$ & $\begin{array}{l}1066 \pm \\
931\end{array}$ & $\begin{array}{l}1482 \pm \\
1501\end{array}$ & $\begin{array}{l}2689 \pm \\
3436\end{array}$ & $\begin{array}{l}1281 \pm \\
1207\end{array}$ \\
\hline & 0.193 & & $0.029^{\ddagger}$ & & 0.163 & & 0.144 & & 0.552 & \\
\hline \multirow[t]{2}{*}{$v_{e}\left(10^{-3}\right)$} & $206 \pm 192$ & $144 \pm 83$ & $\begin{array}{l}286 \pm \\
280\end{array}$ & $155 \pm 97$ & $188 \pm 185$ & $176 \pm 85$ & $\begin{array}{l}239 \pm \\
253\end{array}$ & $156 \pm 79$ & $\begin{array}{l}135 \pm \\
111\end{array}$ & $\begin{array}{l}187 \pm \\
167\end{array}$ \\
\hline & 0.052 & & 0.173 & & 0.722 & & 0.115 & & 0.513 & \\
\hline \multirow[t]{2}{*}{$v_{p}\left(10^{-3}\right)$} & $121 \pm 99$ & $117 \pm 50$ & $114 \pm 74$ & $121 \pm 88$ & $119 \pm 92$ & $120 \pm 60$ & $\begin{array}{l}139 \pm \\
118\end{array}$ & $109 \pm 59$ & $75 \pm 39$ & $121 \pm 86$ \\
\hline & 0.830 & & 0.729 & & 0.984 & & 0.242 & & 0.159 & \\
\hline \multirow[t]{2}{*}{$\mathrm{iAUC}_{60}\left(10^{-3}\right)$} & $378 \pm 412$ & $\begin{array}{l}266 \pm \\
135\end{array}$ & $\begin{array}{l}511 \pm \\
622\end{array}$ & $289 \pm 187$ & $350 \pm 393$ & $307 \pm 127$ & $\begin{array}{l}458 \pm \\
536\end{array}$ & $\begin{array}{l}276 \pm \\
150\end{array}$ & $\begin{array}{l}300 \pm \\
285\end{array}$ & $\begin{array}{l}341 \pm \\
349\end{array}$ \\
\hline & 0.086 & & 0.165 & & 0.471 & & 0.102 & & 0.831 & \\
\hline
\end{tabular}

Abbreviations: MTV = metabolic tumor volume; $S_{U V} V_{\max }=$ maximum standardized uptake value; $k_{\mathrm{ep}}=$ reverse reflux rate constant; $K^{\text {trans }}=$ volume transfer constant; $v_{p}=$ blood plasma volume fraction; $v_{e}=$ extracellular extravascular volume fraction; $A D C=$ apparent diffusion coefficient; iAUC $C_{60}=$ initial area $_{\text {under }}$ the time-tosignal intensity curve at $60 \mathrm{~s}$ post enhancement

* Tumor stage was classified by the American Joint Committee on Cancer 8th edition

\# EGFR mutation was defined as the presence of an EGFR exon19del or L858R mutation in tumor genomic DNA

† Anaplastic lymphoma kinase (ALK)/c-ros oncogene 1 (ROS1) rearrangement was defined as the presence of an ALK or ROS1 rearrangement in tumor genomic DNA

* Significance was tested using Student's t-test

₹ P-values $<0.05$ were considered statistically significant

biomarkers, and radiologic and serum biomarkers were associated with advanced tumor stage. Our findings indicate that patients with higher initial angiogenesisrelatedMR-PET parameters may have benefited from angiogenesis inhibitors, and the imaging biomarkers could be potentially used to guide the clinical use of angiogenesis inhibitors. This study provides a noninvasive, reliable method for the quantitative assessment of tumor angiogenesis in NSCLC patients.
Novel imaging parameters have been demonstrated to be useful in the evaluation of tumor angiogenesis in NSCLC [19]. The ADC value in DW-MRI, indicating the amount of water diffusion within tumors, has been shown to be inversely correlated with tumor angiogenesis, while the parameters MTV and $\mathrm{SUV}_{\max }$ in FDGPET studies correlate with tumor aggressiveness [7, 20]. In our study, image parameters showed significant positive correlations with the concentration of serum 


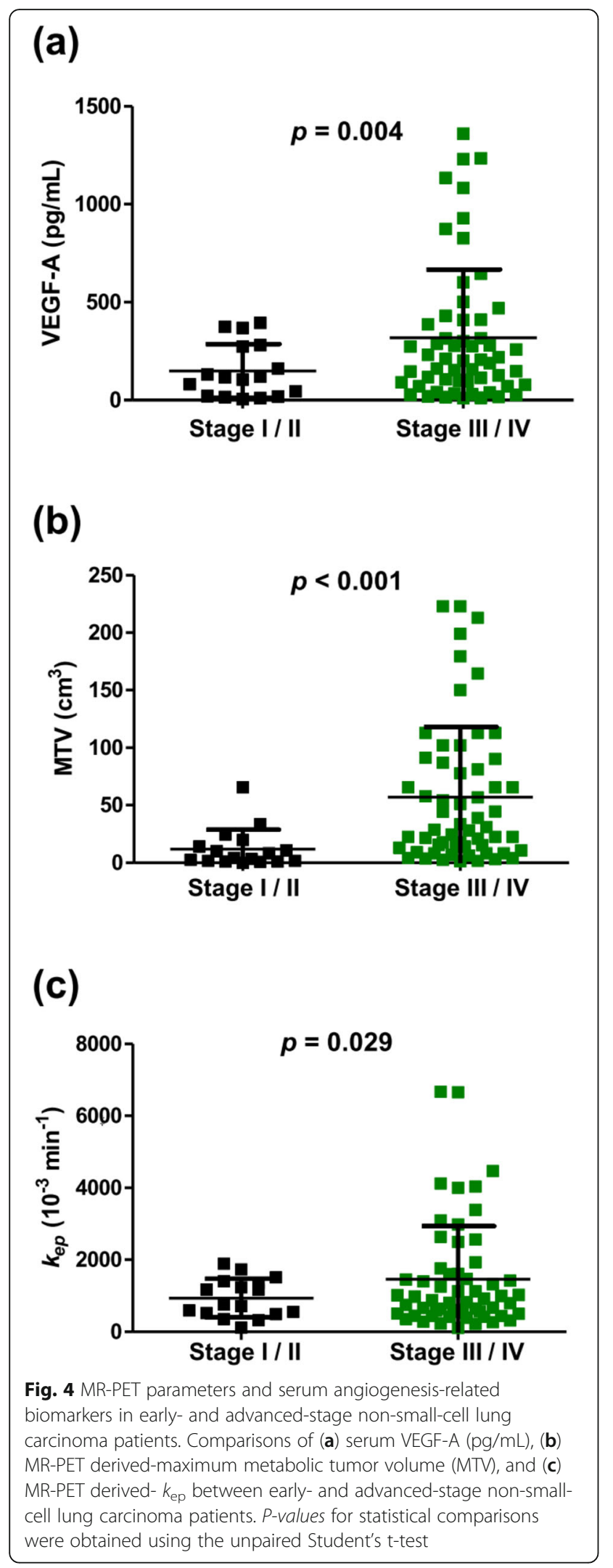

Table 4 Overall survival analysis in advanced-stage patients who received or did not receive angiogenesis inhibitors

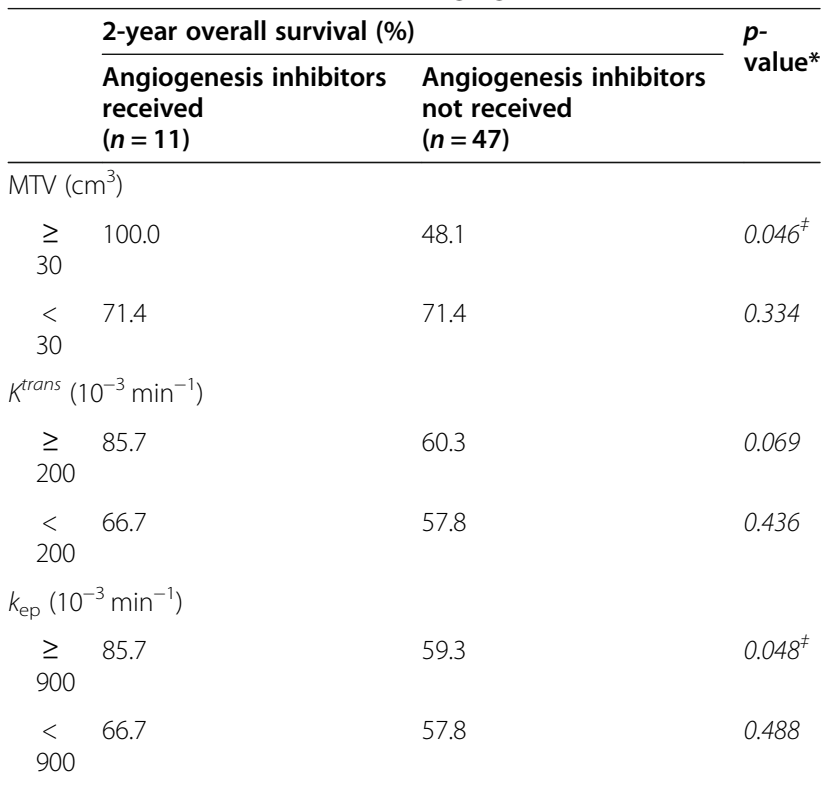

Abbreviations: $\mathrm{MR}-\mathrm{PET}=$ magnetic resonance positron emission tomography; MTV = metabolic tumor volume; $K^{\text {trans }}=$ volume transfer constant; $k_{\mathrm{ep}}=$ reverse reflux rate constant

* Significance was tested using Kaplan-Meier analysis and log-rank tests

${ }^{\ddagger} p$-values $<0.05$ were considered statistically significant

angiogenesis biomarkers, further supporting the application of MR-PET parameters in tumor evaluation [21, 22]. In addition, accumulating studies have examined imaging parameters and serum angiogenesis-related biomarkers in several solid tumors, including NSCLC, rectal cancer, prostate cancer, and breast cancer. For example, Kaira et al. included 37 NSCLC patients and demonstrated positive correlations between IHC VEGF and ${ }^{18}$ F-FDG/PET-derived $\mathrm{SUV}_{\max }[8]$. In patients with rectal cancer, George et al. studied 31 patients and found a positive correlation between serum VEGF and MRderived $K^{\text {trans }}$ [23], Atkin et al. evaluated 15 patients and demonstrated a positive correlation between serum VEGF and IHC microvascular density (MVD) [24], and Yeo et al. analyzed 46 patients and demonstrated a positive correlation between IHC MVD and MR-derived $k_{\mathrm{ep}}$ [25]. In patients with prostate cancer, Oto et al. evaluated 73 men and found a negative correlation between the Gleason score and MR-derived ADC and a positive correlation between IHC MVD and $v_{e}$ [26]. In patients with breast cancer, Kim et al. studied 81 women and found a positive correlation between IHC MVD and $v_{e}$ [27]. These previous findings, which are in line with our results, demonstrate that MR-PET imaging and serum biomarkers can be used to quantitatively assess tumor angiogenesis.

In our study, higher serum and radiologic biomarkers were detected in non-adenocarcinoma tumors than in adenocarcinomas, although the differences were not 
(a)

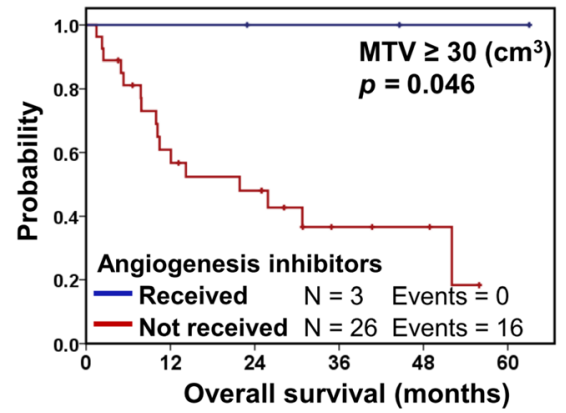

\begin{tabular}{cccccc}
\multicolumn{2}{l}{ No at risk } \\
-3 & 3 & 2 & 2 & 1 & 1 \\
-26 & 14 & 11 & 4 & 3 & 0
\end{tabular}

(c)

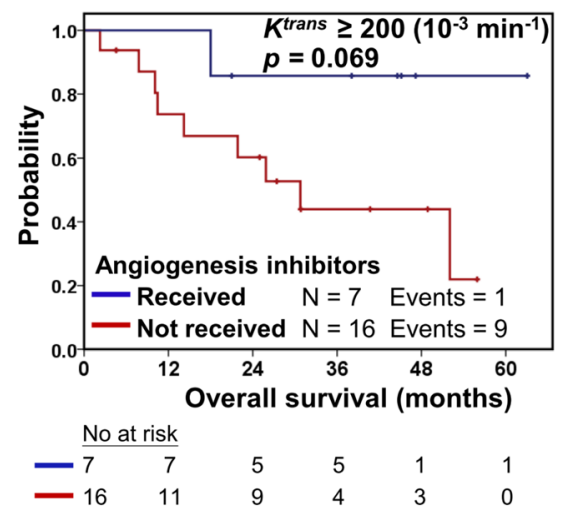

(e)

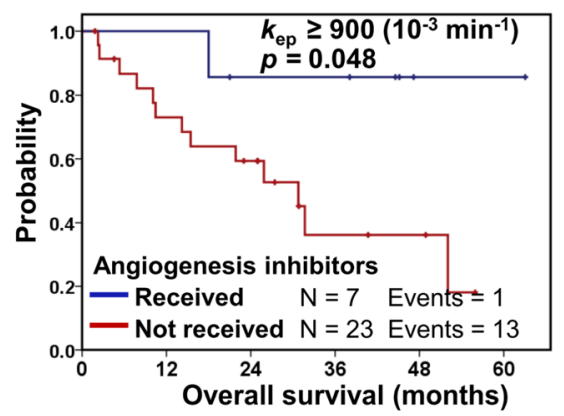

\begin{tabular}{cccccc}
\multicolumn{2}{l}{ No at risk } \\
\hline 7 & 7 & 5 & 5 & 1 & 1 \\
-23 & 16 & 12 & 4 & 3 & 0
\end{tabular} (b)

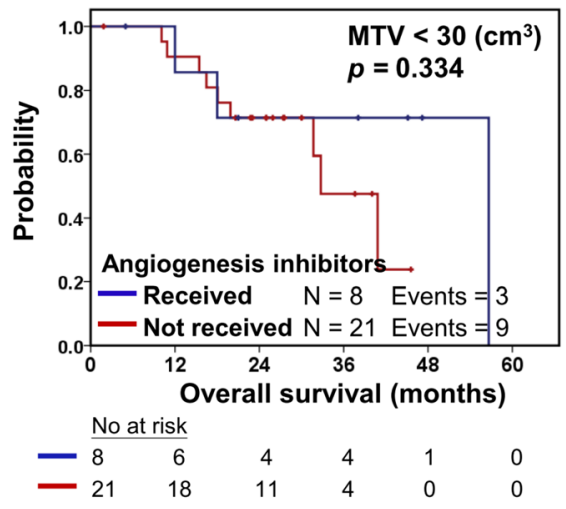

(d)

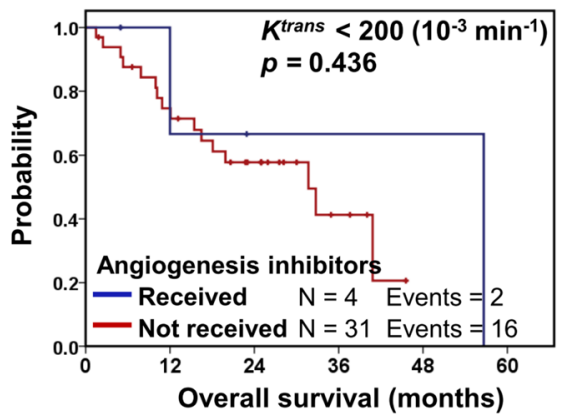

No at risk

\begin{tabular}{llllll}
\hline 4 & 2 & 1 & 1 & 1 & 0 \\
31 & 21 & 13 & 4 & 0 & 0
\end{tabular}

(f)

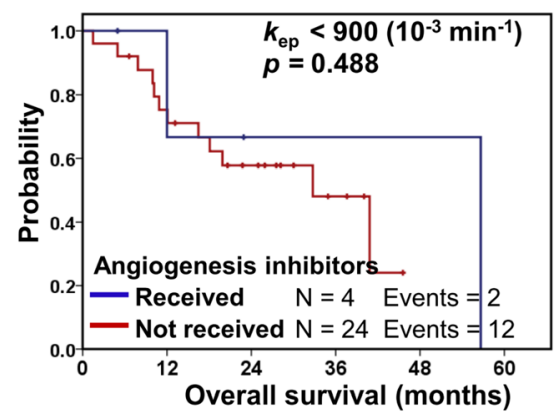

No at risk

$\begin{array}{cccccc}-4 & 2 & 1 & 1 & 1 & 0 \\ -24 & 17 & 11 & 4 & 0 & 0\end{array}$

Fig. 5 Overall survival analysis in advanced-stage patients who received or did not receive angiogenesis inhibitors. Patients were grouped according to initial angiogenesis-related MR-PET parameters, i.e., (a) metabolic tumor volume (MTV) $\geq 30$ or (b) $<30 \mathrm{~cm}^{3}$, (c) $K^{\text {trans }} \geq 200$ or (d) $<$ $200\left(10^{-3} \mathrm{~min}^{-1}\right)$, and $(\mathbf{e}) k_{\mathrm{ep}} \geq 900$ or $(\mathbf{f})<900\left(10^{-3} \mathrm{~min}^{-1}\right)$. P-values were determined using Kaplan-Meier log-rank tests

statistically significant. These findings can be partly explained by the higher proportion of advanced-stage (stage III or IV) tumors in the non-adenocarcinoma group than in the adenocarcinoma group ( $89 \%$ vs. $73 \%$, $p<0.001)$. Consistently, previous studies also showed that non-adenocarcinoma tumors had higher SUV and Ki67 scores and were characterized by higher tumor aggressiveness [28, 29]. Whether non-adenocaricnoma 
tumors poccessed with dissimilar proliferation pattern, glucose metabolism, or aggressiveness than adenocarcinoma tumors need further investigation.

Our study showed that patients with higher initial angiogenesis-relatedMR-PET parameters (including MTV $>30 \mathrm{~cm}^{3}, \quad K^{\text {trans }}>20010^{-3} / \mathrm{min}$, and $k_{\text {ep }}>900$ $10^{-3} / \mathrm{min}$ ) may have benefited from angiogenesis inhibitors, resulting in longer survival. Consistently, de Langen et al. demonstrated that patients with a significant decrease in SUV or tumor perfusion three weeks post bevacizumab and erlotinib treatment had longer survival [12], and Kelly et al. demonstrated correlations between changes in $k_{\text {ep }}$ and serum basic fibroblast growth factor and progression-free survival in patients who received sorafenib [13]. The above-mentioned findings, in line with our results, further demonstrate radiological and cytokine changes as biomarkers indicative of early angiogenesis inhibition, and these biomarkers can be used to identify patients who may benefit from angiogenesis inhibitors.

Novel PET radiopharmaceuticals for imaging angiogenesis are under investigation in lung cancer patients, and $\alpha_{v} \beta_{3}$ integrin, which upregulates activated neovascular endothelial cells in association with tumor angiogenesis, has been targeted for PET imaging [30]. Arg-GlyAsp (RGD) peptide-based PET tracers, which have been developed to image integrin expression in tumors and are predominantly used in preclinical environment with clinical implementation, are being studied at present [31, 32]. Since the correlation of endothelial integrin and glucose metabolism in malignant lesions needs further assessment, preliminary results from novel PET radiopharmaceuticals warrant attentive interpretation for response evaluation for targeted molecular therapies with antiangiogenic or integrin-targeted agents.

Our study has several limitations. A total of 75 NSCL $\mathrm{C}$ patients and 15 healthy controls were included in the study; the number of patients and controls are not balanced. However, since the study was not a case-control study or paired comparison analysis, and unpaired Student's t-tests were performed for statistical analysis between patients and controls, the imbalance does not influence the accuracy of our data interpretation. Serum angiogenesis biomarkers are thought to represent the average concentrations secreted by all tumors in the body, including the main tumor; thus, the serum biomarker concentrations might indicate integral tumor aggressiveness but might not comprehensively reflect individual tumor heterogeneity.

DCE-integrated MR-PET imaging presents as being a promising non-invasive method for assessing tumor angiogenesis in patients with NSCLC. Our findings suggest that DCE-integratedMR-PET imaging may be useful for assessing angiogenesis in patients with NSCLC at diagnosis, identifying patients who may benefit from being treated with angiogenesis inhibitors, and monitoring the response to therapies.

\section{Conclusions}

Radiologic parameters derived from DCE-integratedMRPET scans correlated with serum angiogenesis-related biomarkers in NSCLC patients and could be used to potentially guide the clinical use of angiogenesis inhibitors. Since tumor angiogenesis is an important prognostic factor for anticancer treatment and patient survival, our results suggest that DCE-integratedMR-PET imaging, which provides a non-invasive, quantitative assessment of tumor angiogenesis, may play a role in personalized medicine for patients with NSCLC.

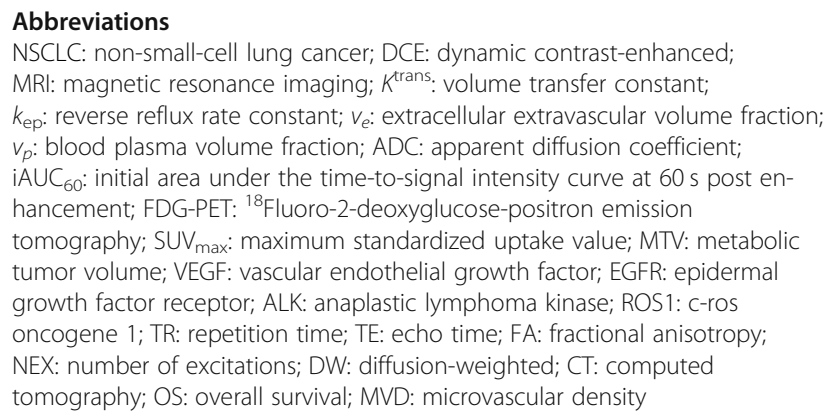

\section{Acknowledgments}

We thank Dr. Tzu-Hsiu Tsai, Dr. Chia-Lin Hsu, Dr. Yen-Ting Lin, Dr. Ching-Yao Yang, and Prof. Chao-Chi Ho for assisting with this study. We are thankful to the staff of the Core Labs, Department of Medical Research, National Taiwan University Hospital for their technical support.

\section{Authors' contributions}

$Y H, J C, H C, L Y$, and $Y C$ performed the experiments. $Y H, J C, J S, R Y$, and $Y C$ analyzed and interpreted the data. $\mathrm{YH}, \mathrm{JC}$, and $\mathrm{YC}$ were major contributors in writing the manuscript. JS and RY supervised the study, reviewed and edited the manuscript. All authors read and approved the final manuscript.

\section{Funding}

This work was supported by the Ministry of Science and Technology Taiwan (grant number: Contract No. MST 104-2221-E-002-093-MY3, 106-2221-E-002081-MY3, and 107-2314-B-002-225-MY2) and National Taiwan University Hospital (grant number: NTUH106-N3662). The funding bodies played no role in the design of the study and collection, analysis, and interpretation of data and in writing the manuscript.

\section{Availability of data and materials}

The datasets used and/or analyzed during the current study are available from the corresponding author on reasonable request.

\section{Declarations}

Ethics approval and consent to participate

This prospective study was conducted according to the guidelines of the Declaration of Helsinki and its later amendments, and was approved by the National Taiwan University Hospital Research Ethics Committee (approval number: 201712101RIND). Written informed consent was obtained from all participants after the nature of the procedures had been fully explained.

Consent for publication

Not applicable. 


\section{Competing interests}

The authors declare that they have no competing interests.

\section{Author details}

'Department of Radiology, National Taiwan University College of Medicine, No. 7, Chung-Shan S. Rd., Taipei 100, Taiwan. ${ }^{2}$ Department of Medical Imaging, National Taiwan University Hospital, Taipei, Taiwan. ${ }^{3}$ Department of Oncology, National Taiwan University Hospital, Taipei, Taiwan. ${ }^{4}$ National Taiwan University Cancer Center, National Taiwan University College of Medicine, Taipei, Taiwan. ${ }^{5}$ Department of Internal Medicine National Taiwan University Hospital, Taipei, Taiwan. ${ }^{6}$ Department of Nuclear Medicine, National Taiwan University Hospital, Taipei, Taiwan.

\section{Received: 5 November 2020 Accepted: 18 March 2021} Published online: 01 April 2021

\section{References}

1. Hall RD, Le TM, Haggstrom DE, Gentzler RD. Angiogenesis inhibition as a therapeutic strategy in non-small cell lung cancer (NSCLC). Translational Lung Cancer Res. 2015:4(5):515-23.

2. Lee SN. The prognostic role of vascular endothelial growth factor (VEGF) expression and angiogenesis in curatively resected non-small cell lung cancer. Cancer Res Treat. 1999;31(6):1210-8.

3. Chen JL, Pan CK, Huang YS, Tsai CY, Wang CW, Lin YL, et al. Evaluation of antitumor immunity by a combination treatment of high-dose irradiation, anti-PDL1, and anti-angiogenic therapy in murine lung tumors. Cancer Immunol Immunother. 2021;70(2):391-404. https://doi.org/10.1007/s00262020-02690-w.

4. Huang YS, Chen JL, Hsu FM, Huang JY, Ko WC, Chen YC, et al. Response assessment of stereotactic body radiation therapy using dynamic contrastenhanced integrated MR-PET in non-small cell lung cancer patients. J Magn Reson Imaging. 2018;47(1):9.

5. Nielsen T, Wittenborn T, Horsman MR. Dynamic contrast-enhanced magnetic resonance imaging (DCE-MRI) in preclinical studies of antivascular treatments. Pharmaceutics. 2012;4(4):563-89. https://doi.org/10.3390/pharma ceutics4040563.

6. Chen HH, Chiu NT, Su WC, Guo HR, Lee BF. Prognostic value of whole-body total lesion glycolysis at pretreatment FDG PET/CT in non-small cell lung cancer. Radiology. 2012;264(2):559-66. https://doi.org/10.1148/ra diol.12111148.

7. Berghmans T, Dusart M, Paesmans M, Hossein-Foucher C, Buvat I, Castaigne $C$, et al. Primary tumor standardized uptake value (SUVmax) measured on fluorodeoxyglucose positron emission tomography (FDG-PET) is of prognostic value for survival in non-small cell lung cancer (NSCLC): a systematic review and meta-analysis (MA) by the European lung Cancer working party for the IASLC lung Cancer staging project. J Thorac Oncol. 2008;3(1):6-12. https://doi.org/10.1097/JTO.0b013e31815e6d6b.

8. Kaira K, Oriuchi N, Shimizu K, Ishikita T, Higuchi T, Imai H, et al. Correlation of angiogenesis with 18F-FMT and 18F-FDG uptake in non-small cell lung cancer. Cancer Sci. 2009;100(4):753-8. https://doi.org/10.1111/j.1349-7006.2 008.01077.x.

9. Laack E, Scheffler A, Burkholder I, Boeters I, Andritzky B, Schuch G, et al. Pretreatment vascular endothelial growth factor (VEGF) and matrix metalloproteinase-9 (MMP-9) serum levels in patients with metastatic nonsmall cell lung cancer (NSCLC). Lung Cancer. 2005;50(1):51-8. https://doi. org/10.1016/j.lungcan.2005.05.011.

10. Majeti BK, Lee JH, Simmons BH, Shojaei F. VEGF is an important mediator of tumor angiogenesis in malignant lesions in a genetically engineered mouse model of lung adenocarcinoma. BMC Cancer. 2013;13(1):213. https://doi. org/10.1186/1471-2407-13-213

11. Tello-Montoliu A, Patel JV, Lip GY. Angiogenin: a review of the pathophysiology and potential clinical applications. J Thromb Haemost. 2006:4(9):1864-74. https://doi.org/10.1111/j.1538-7836.2006.01995.x.

12. de Langen AJ, van den Boogaart $\mathrm{V}$, Lubberink M, Backes WH, Marcus JT, van Tinteren $\mathrm{H}$, et al. Monitoring response to antiangiogenic therapy in nonsmall cell lung cancer using imaging markers derived from PET and dynamic contrast-enhanced MRI. J Nucl Med. 2011;52(1):48-55. https://doi. org/10.2967/jnumed.110.078261.

13. Kelly RJ, Rajan A, Force J, Lopez-Chavez A, Keen C, Cao L, et al. Evaluation of KRAS mutations, angiogenic biomarkers, and DCE-MRI in patients with advanced non-small-cell lung cancer receiving sorafenib. Clin Cancer Res. 2011;17(5):1190-9. https://doi.org/10.1158/1078-0432.CCR-10-2331.

14. Kay FU, Kandathil A, Batra K, Saboo SS, Abbara S, Rajiah P. Revisions to the tumor, node, metastasis staging of lung cancer (8(th) edition): rationale, radiologic findings and clinical implications. World J Radiol. 2017;9(6):26979. https://doi.org/10.4329/wjr.v9.i6.269.

15. Yao ZH, Liao WY, Ho CC, Chen KY, Shih JY, Chen JS, et al. Real-world data on prognostic factors for overall survival in egfr mutation-positive advanced non-small cell lung cancer patients treated with first-line gefitinib. Oncologist. 2017;22(9):1075-83. https://doi.org/10.1634/theoncologist.20160331

16. Huang YS, Chen JL, Chen JY, Lee YF, Huang JY, Kuo SH, et al. Predicting tumor responses and patient survival in chemoradiotherapy-treated patients with non-small-cell lung cancer using dynamic contrast-enhanced integrated magnetic resonance-positron-emission tomography. Strahlenther Onkol. 2019;195(8):707-18. https://doi.org/10.1007/s00066-018-1418-8.

17. Chen JL, Chang CC, Huang YS, Kuo HY, Chen TY, Wang CW, et al. Persistently elevated soluble MHC class I polypeptide-related sequence a and transforming growth factor-beta1 levels are poor prognostic factors in head and neck squamous cell carcinoma after definitive chemoradiotherapy. PLoS One. 2018;13(8):e0202224. https://doi.org/10.1371/ journal.pone.0202224.

18. Gates S. Statistical significance and clinical evidence. Lancet Oncol. 2020; 21(3):e118. https://doi.org/10.1016/S1470-2045(19)30854-X.

19. Ma SH, Le HB, Jia BH, Wang ZX, Xiao ZW, Cheng XL, et al. Peripheral pulmonary nodules: relationship between multi-slice spiral CT perfusion imaging and tumor angiogenesis and VEGF expression. BMC Cancer. 2008; 8(1):186. https://doi.org/10.1186/1471-2407-8-186.

20. Kwak Y-K, Park HH, Choi KH, Park EY, Sung SY, Lee S-W, et al. SUVmax predicts disease progression after stereotactic ablative radiotherapy in stage i non-small cell lung cancer. Cancer Res Treat. 2020;52(1):85-97. https://doi. org/10.4143/crt.2019.007.

21. O'Connor JP, Jayson GC. Do imaging biomarkers relate to outcome in patients treated with VEGF inhibitors? Clin Cancer Res. 2012;18(24):6588-98. https://doi.org/10.1158/1078-0432.CCR-12-1501.

22. O'Connor JPB, Jackson A, Parker GJM, Roberts C, Jayson GC. Dynamic contrast-enhanced MRI in clinical trials of antivascular therapies. Nat Rev Clin Oncol. 2012;9(3):167-77. https://doi.org/10.1038/nrclinonc.2012.2.

23. George ML, Dzik-Jurasz AS, Padhani AR, Brown G, Tait DM, Eccles SA, et al. Non-invasive methods of assessing angiogenesis and their value in predicting response to treatment in colorectal cancer. Br J Surg. 2001;88(12): 1628-36. https://doi.org/10.1046/j.0007-1323.2001.01947.x.

24. Atkin G, Taylor NJ, Daley FM, Stirling JJ, Richman P, Glynne-Jones R, et al. Dynamic contrast-enhanced magnetic resonance imaging is a poor measure of rectal cancer angiogenesis. Br J Surg. 2006;93(8):992-1000. https://doi.org/10.1002/bjs.5352.

25. Yeo DM, Oh SN, Jung CK, Lee MA, Oh ST, Rha SE, et al. Correlation of dynamic contrast-enhanced MRI perfusion parameters with angiogenesis and biologic aggressiveness of rectal cancer: preliminary results. J Magn Reson Imaging. 2015;41(2):474-80. https://doi.org/10.1002/jmri.24541.

26. Oto A, Yang C, Kayhan A, Tretiakova M, Antic T, Schmid-Tannwald C, et al. Diffusion-weighted and dynamic contrast-enhanced MRI of prostate cancer: correlation of quantitative MR parameters with Gleason score and tumor angiogenesis. AJR Am J Roentgenol. 2011;197(6):1382-90. https://doi.org/1 $0.2214 / A J R .11 .6861$

27. Kim JY, Kim SH, Kim YJ, Kang BJ, An YY, Lee AW, et al. Enhancement parameters on dynamic contrast enhanced breast MRI: do they correlate with prognostic factors and subtypes of breast cancers? Magn Reson Imaging. 2015;33(1):72-80. https://doi.org/10.1016/j.mri.2014.08.034.

28. Vesselle H, Salskov A, Turcotte E, Wiens L, Schmidt R, Jordan CD, et al. Relationship between non-small cell lung cancer fdg uptake at pet, tumor histology, and ki-67 proliferation index. J Thorac Oncol. 2008;3(9):971-8. https://doi.org/10.1097/JTO.0b013e31818307a7.

29. Lohith TG, Kudo T, Demura Y, Umeda Y, Kiyono Y, Fujibayashi Y, et al. Pathophysiologic correlation between 62CU-ATSM and 18F-FDG in lung cancer. J Nucl Med. 2009;50(12):1948-53. https://doi.org/10.2967/jnumed.109.069021.

30. Szyszko TA, Yip C, Szlosarek P, Goh V, Cook GJR. The role of new PET tracers for lung cancer. Lung Cancer. 2016;94:7-14. https://doi.org/10.1016/j.lungca n.2016.01.010

31. Kenny LM, Coombes RC, Oulie I, Contractor KB, Miller M, Spinks TJ, et al. Phase I trial of the positron-emitting Arg-Gly-asp (RGD) peptide radioligand 
18F-AH111585 in breast cancer patients. J Nucl Med. 2008;49(6):879-86. https://doi.org/10.2967/jnumed.107.049452.

32. Beer AJ, Lorenzen S, Metz S, Herrmann K, Watzlowik P, Wester HJ, et al. Comparison of integrin av $\beta 3$ expression and glucose metabolism in primary and metastatic lesions in cancer patients: a PET study using 18F-GalactoRGD and 18F-FDG. J Nucl Med. 2008;49(1):22-9. https://doi.org/10.2967/ jnumed.107.045864.

\section{Publisher's Note}

Springer Nature remains neutral with regard to jurisdictional claims in published maps and institutional affiliations.

Ready to submit your research? Choose BMC and benefit from:

- fast, convenient online submission

- thorough peer review by experienced researchers in your field

- rapid publication on acceptance

- support for research data, including large and complex data types

- gold Open Access which fosters wider collaboration and increased citations

- maximum visibility for your research: over $100 \mathrm{M}$ website views per year

At $\mathrm{BMC}$, research is always in progress.

Learn more biomedcentral.com/submissions 